\title{
Digital Two-Degree-of-Freedom Controller for Processes with Large Time-delay
}

\author{
B. Del MURO CUÉLLAR ${ }^{1}$, R.J. VAZQUEZ GUERRA²*, J.F. MÁRQUEZ RUBIO ${ }^{1}$ \\ ${ }^{1}$ Instituto Politécnico Nacional, ESIME Unidad Culhuacán, Av. Santa Ana 1000, \\ Coyoacán, 04430, CDMX, México \\ bdelmuro@yahoo.com,jfcomr23@yahoo.com.mx \\ ${ }^{2}$ Tecnologico de Monterrey, Escuela de Ingeniería y Ciencias, \\ Ave. Eugenio Garza Sada 2501, Monterrey, N.L., México \\ rjjvg@tec.mx (*Corresponding author)
}

\begin{abstract}
In this work a methodology for the stabilization and control of processes with large time-delay is proposed. This methodology considers a digital controller with two degrees of freedom, which is tuned via the polynomial approach. The proposed digital controller is able to deal with linear time invariant systems, delayed input/output systems i.e., stable, unstable and integrating delayed processes. The problems of step reference tracking and step disturbance rejection are also addressed. Finally, numerical simulations are used for illustrating the performance of the proposed control strategy in comparison with recent proposals in literature.
\end{abstract}

Keywords: Systems with time-delays, Time-invariant systems, Stabilization, Unstable processes, Process control.

\section{Introduction}

Time-delay systems (also called dead-time systems) are commonly found in industrial processes (Richard, 2003). Modeling of chemical processes, production chains, etc., can induce timedelays. Also, this delay term can be introduced by the physical properties of the equipment involved, when transporting some kind of mass or energy (distillation columns, heat exchange processes, etc.), by the measurement of the variables of the system, by the time consumed in computing the control law and so on (Liu, Zhang \& Gu, 2005). Time-delay systems are challenging from the viewpoint of control and they should be approached through control strategies with an acceptable performance and closed-loop stability. Several control strategies have been developed in order to deal with time delay systems. The Smith Predictor Compensator (SPC) has been widely used as a prediction strategy (Owens \& Raya, 1982; Palmor, 1996). However, the SPC can only be applied to stable processes. In order to overcome this limitation, some modifications to the original SPC structure have been proposed for unstable processes (Márquez-Rubio et al., 2015; Fragoso-Rubio et al. 2019). For example, in (Márquez-Rubio et al., 2015) an observerbased approach to first-order unstable systems is presented, where the stability condition is stated in terms of the delay size related to the inverse of the dominant pole position in unstable process. Such an approach has reported time delays as large as four times the unstable time constant of the open-loop system. Also certain extensions of the SPC to control processes with one integrating and large time-delay have been reported (García \& Albertos, 2008; Visioli \& Zhong, 2011). In (García \& Albertos, 2013) a Generalized Predictor (GP) has been presented to control stable, integral and unstable delayed processes. This GP considers a discrete approach and proposes a predictorbased control structure with two filters and a PID controller. On the other hand, the classical PI/ PID controllers have also been employed with a view to controlling delayed systems, namely for integrating and unstable processes with time-delay (Seshagiri \& Chidambaram, 2012; Gang-Gyoo \& Yug-Deug, 2019). These control strategies perform acceptably in the control response when the delay is relatively small when compared to the unstable time constant of the system (Hagglund, 1996).

In this work a discrete control methodology is presented in order to deal with systems with large time-delays. The proposed control structure is based on a polynomial approach and on a twodegree-of-freedom controller. One important contribution of this work lies in the simplicity of the proposed methodology, since this new proposal does not consider any prediction stage to design the control law. This methodology uses a discrete time approach to tune the two-degree-offreedom controller, which is able to improve the closed-loop performance with respect to previous works in literature (García \& Albertos, 2013; García \& Albertos, 2008).

The above-mentioned control strategy is also compared with the prediction-observer approach presented in a recent work (Fragoso-Rubio et 
al. 2019). Additionally, this paper considers the problems of step tracking reference and the step disturbance rejection. This paper is organized as follows. In Section 2 the problem statement is presented. Section 3 sets forth the main results obtained. Further on, the numerical simulations in Section 4 illustrate the performance of the aforementioned methodology. Finally, the conclusion of this paper is included in Section 5.

\section{Problem Statement}

Let us consider the following class of Linear Time Invariant (LTI), Single-Input Single-Output (SISO) systems with delay at the input/output path

$$
\frac{Y(s)}{U(s)}=\frac{N(s)}{M(s)} e^{-\tau s}=G(s) e^{-\tau s},
$$

where $U(s)$ and $Y(s)$ are the input and output signals, respectively; $\tau \geq 0$ is the constant timedelay; $N(s)$ and $M(s)$ are polynomials of the complex variable $s$, and $G(s)$ is the delay-free transfer function. Consider a traditional control strategy based on an output feedback

$U(s)=[R(s)-Y(s)] F(s)$,

which yields to the closed-loop system given by

$$
\frac{Y(s)}{R(s)}=\frac{F(s) G(s) e^{-\tau s}}{1+F(s) G(s) e^{-\tau s}},
$$

where the exponential term $\left(e^{-\tau s}\right)$ located in the characteristic equation of the transfer function (2) leads to a system with an infinite number of poles. Then, the properties pertaining to closedloop stability should be carefully stated.

The stability analysis is complicated due to the delay term. In this proposal a discrete time representation of the time-delay term is considered. This consideration allows to obtain a controller tuned via polynomial approach.

\section{Main Results}

In this section a digital control methodology for stable, integrating and unstable systems with large time-delay is presented. In this methodology the time-delay term is expressed by a multiple of the sampling period $T=\frac{\tau}{n}$, where $\tau$ is the time-delay and $n \in \mathrm{N}$. This consideration on the sampling period was also used in (García \& Albertos, 2013) in order to obtain a rational transfer function representation into variable $z$ domain (without transcendental terms). In the present work such a sampling period is taken into account with the same intention. Based on this assumption, let us consider the discrete time representation of (1),

$\frac{Y(z)}{U(z)}=G_{d}(z)$

where $G_{d}$ represents the z-transform of (1), for a zero-order hold $(\mathrm{ZOH})$. Therefore, the transfer function $G_{d}(z)$, can also be expressed as

$G_{d}(z)=\left(\frac{N(z)}{M(z)}\right) z^{-n}=\frac{B(z)}{A(z)}$

It should be noticed that the transfer function $G_{d}(z)$ includes the terms of the delay-free system $\frac{N(z)}{M(z)}$ and the (large) delay, which is expressed as $n$ unitary delays $\left(z^{-n}\right)$. It should be taken into account that the discrete representation of the delay operator increases the relative degree of the whole discrete process representation due to the chosen sampling period. The following subsection presents the procedure employed in order to design the proposed discrete controller with two-degreeof-freedom via the polynomial approach.

\subsection{Control Structure}

Consider the discrete representation of the process equation (3), which can be rewritten as

$G_{d}(z)=\frac{Y(z)}{U(z)}=\frac{B(z)}{A(z)}$,

where $B(z)$ and $A(z)$ are polynomials of the complex variable $z . U(z)$ and $Y(z)$ are the input and the measurable digital output signals, respectively. The proposed two-degree-offreedom controller can be represented as follows:

$U(z)=-\frac{R(z)}{S(z)} Y(z)+\frac{T(z)}{S(z)} Y_{c}(z)$

where $Y_{c}(z)$ is the new reference input variable. The proposed digital controller structure with twodegree-of-freedom is illustrated in Figure 1. The transfer functions $\frac{T(z)}{S(z)}$ and $\frac{R(z)}{S(z)}$ are considered to be strictly proper. By substituting the control law given by (5) into transfer function (4), the following closed-loop transfer function is obtained,

$$
\frac{Y(z)}{Y_{c}(z)}=\frac{B(z) T(z)}{A(z) S(z)+B(z) R(z)} .
$$




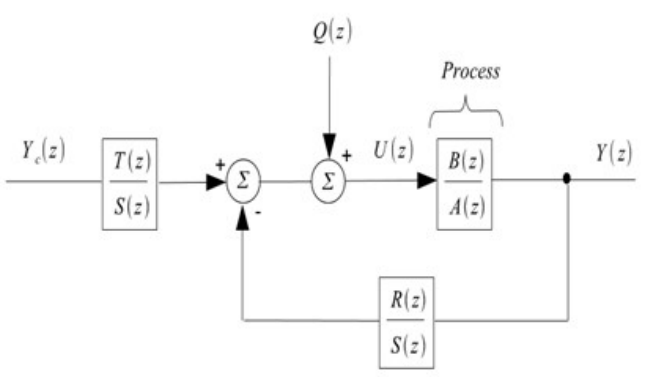

Figure 1. Digital controller with two-degrees-offreedom and disturbance input

The characteristic equation induced by the digital controller is given by

$$
A(z) S(z)+B(z) R(z)=0 .
$$

It should be noticed that one has complete freedom to design the polynomials $S(z)$ and $R(z)$ in equation (7), thus the closed-loop poles can be relocated freely. However, the dimension of such polynomials should be established adequately. In that case, the characteristic polynomial (7) should be equaled with a desired characteristic stable polynomial $D(z)$, where $D(z)$ should be of the same order as (7),

$$
D(z)=A(z) S(z)+B(z) R(z) \text {. }
$$

It can be seen that the output response of the system depends on $D(z)$. The desired closedloop poles must be relocated into the unitary circle in the complex plane $z$ in order to obtain a stable response. It is recommended to place the poles on the interval $[0,1)$, into the real axis in order to obtain a transient overdamped output response i.e., without transient oscillation performance. On the other hand the control parameters $S(z)$ and $R(z)$ can be calculated by solving a set of algebraic equations, which are derived from equation (8). The details for solving the induced system of equations will be provided later.

The proposed methodology can be applied for systems without delay by considering a discrete representation (3) with $\mathrm{n}=0$ and using any sample period $\mathrm{T}$ defined for the user.

\subsection{Step Disturbance Rejection}

In order to analyse the disturbance rejection problem, let us consider the disturbed process input

$U(z)=-\frac{R(z)}{S(z)} B(z)+Q(z)$,

where $Q(z)$ is a step input disturbance. By substituting expression (9) into transfer function
(4), the disturbance-output transfer function can be obtained:

$$
\frac{Y(z)}{Q(z)}=\frac{S(z) B(z)}{A(z) S(z)+B(z) R(z)} \cdot
$$

Figure 1 illustrates the digital controller with two-degree-of-freedom and disturbance input $Q(z)$. The classical Final Value Theorem can be applied to $Y(z)$ derived from transfer function (10), when the disturbance step signal is given by $Q(z)=\frac{z \beta}{(z-1)}$, with an amplitude $\beta \in \mathrm{R}$. The following expression is obtained,

$\lim _{k \rightarrow \infty} y(k)=\lim _{z \rightarrow 1}(z-1)\left(\frac{S(z) B(z)}{A(z) S(z)+B(z) R(z)}\right) Q(z)$

It can be easily shown that when the polynomial $S(z)$ is chosen as $S(z)=(z-1) C^{\prime}(z)$, where $C^{\prime}(z)$ is a new polynomial with degree $\operatorname{deg}(S(z))-1$. Then, $S(1)=0$, i.e., the output $Y(z)$ is zero for the steady-state of the system. Therefore, the system is able to reject input step disturbances. Then, one can present the following property of the proposed methodology.

Property 1. The control scheme shown in Figure 1 is able to reject input step disturbance, if the polynomial $S(z)$ is given by

$S(z)=(z-1) C^{\prime}(z)$

where $C^{\prime}(z)$ is a new polynomial with the degree $\operatorname{deg}(S(z))-1$.

\subsection{Step Reference Tracking}

The following analysis considers the problem of step reference tracking. Let us consider the transfer function given by equation (6). By applying the Final Value Theorem with $Y_{c}=\frac{z \beta}{(z-1)},(\beta \in \mathrm{R})$ as the input reference, to the transfer function given by (6), one obtains:

$\lim _{k \rightarrow \infty} y(k)=\lim _{z \rightarrow 1}(z-1)\left(\frac{B(z) T(z)}{A(z) S(z)+B(z) R(z)}\right) Y_{c}(z)$

Considering the polynomial $S(z)$ given by (11), the limit given by (12) becomes,

$\lim _{z \rightarrow 1}\left(\frac{T(z)}{R(z)}\right) \beta$.

Notice that in the limit given by (13), $T(z)$ should be equal to the sum of the $R(z)$ coefficients in order to track step references. $T(z)$ also can be also expressed as

$T(z)=r_{0}+r_{1}+\ldots+r_{\alpha}$. 
Therefore, the second property of the proposed methodology can be stated as:

Property 2. The control scheme shown in Figure 1 is able to track reference step changes, if $T(z)$ is considered as

$T(z)=R(1)$.

\subsection{Controllers Degree}

Considering the previous developments, the degrees of the polynomials satisfying step disturbance rejection and step reference tracking are given by

$$
\begin{gathered}
\operatorname{deg}(D(z))=2 \operatorname{deg}(A(z))+1 \\
\operatorname{deg}(R(z))=\operatorname{deg}(A(z)), \\
\operatorname{deg}(S(z))=\operatorname{deg}(A(z))+1
\end{gathered}
$$

where the corresponding coefficients are defined as,

$$
\begin{gathered}
D(z)=d_{0} z^{2 \alpha+1}+d_{1} z^{2 \alpha}+\ldots+d_{2 \alpha+1} \\
R(z)=r_{0} z^{\alpha}+r_{1} z^{\alpha-1}+\ldots+r_{\alpha} \\
S(z)=(z-1) C^{\prime}(z), \\
S(z)=(z-1)\left(s_{0} z^{\alpha}+s_{1} z^{\alpha-1}+\ldots+s_{\alpha}\right) \\
T(z)=r_{0}+r_{1}+\ldots+r_{\alpha}
\end{gathered} .
$$

The degrees of $D(z), R(z), S(z)$ and $T(z)$ depend on $\alpha=\operatorname{deg}(A) z$ ). The values of the coefficients $R(z), S(z)$ and $T(z)$ can be obtained by solving a system of simultaneous linear equations. Further on, a method employed with a view to generalizing the proposed discrete polynomial control is presented.

\subsection{General Discrete Polynomial Equation.}

In this section the coefficients of the polynomial controllers $S(z)$ and $R(z)$ are obtained. Equation (8) is known as the Bezout identity and the solutions for $S(z)$ and $R(z)$ are guaranteed. By substituting equation (15) into equation (8), equation (8) can be expressed as a matrix representation,

$M x=d$,

where $M$ is a square matrix of dimension $(2 \operatorname{deg}(A(z))+2) . M$ consists in the known coefficients of polynomials $A(z)$ and $B(z)$, given by the model process (3). $X$ is column vector, which contains the unknown coefficients $S(z)$ and $R(z)$. And finally, $d$ is a known vector with the coefficients of the desired polynomial characteristic $D(z)$. Thus, the coefficients of the polynomials $A(z)$ and $B(z)$ can be generalized as follows:

$$
\begin{gathered}
A(z)=z^{\alpha}+a_{1} z^{\alpha-1}+a_{2} z^{\alpha-2}+\ldots+a_{\alpha}, \\
B(z)=b_{1} z^{\alpha-1}+b_{2} z^{\alpha-2}+\ldots+b_{\alpha}
\end{gathered}
$$

such that the equation (16) is expressed by,

$M=\left[\begin{array}{ll}M_{11} & M_{12}\end{array}\right]$

$M_{11}=\left[\begin{array}{cccccc}1 & 0 & 0 & 0 & 0 & 0 \\ a_{1}-1 & 1 & 0 & 0 & 0 & 0 \\ a_{2}-a_{1} & a_{1}-1 & \ddots & 0 & 0 & 0 \\ a_{3}-a_{2} & a_{2}-a_{1} & \ddots & \ddots & 0 & 0 \\ \vdots & a_{3}-a_{2} & \ddots & \ddots & 1 & 0 \\ a_{\alpha}-a_{\alpha-1} & \vdots & \ddots & \ddots & a_{1}-1 & 1 \\ -a_{\alpha} & a_{\alpha}-a_{\alpha-1} & \vdots & \ddots & a_{2}-a_{1} & a_{1}-1 \\ 0 & -a_{\alpha} & \ddots & \vdots & a_{3}-a_{2} & a_{2}-a_{1} \\ 0 & 0 & \ddots & \ddots & \vdots & a_{3}-a_{2} \\ 0 & 0 & 0 & \ddots & a_{\alpha}-a_{\alpha-1} & \vdots \\ 0 & 0 & 0 & 0 & -a_{\alpha} & a_{\alpha}-a_{\alpha-1} \\ 0 & 0 & 0 & 0 & 0 & -a_{\alpha}\end{array}\right]$

$M_{12}=\left[\begin{array}{cccccc}0 & 0 & 0 & 0 & 0 & 0 \\ 0 & 0 & 0 & 0 & 0 & 0 \\ b_{1} & 0 & \ddots & 0 & 0 & 0 \\ b_{2} & b_{1} & \ddots & \ddots & 0 & 0 \\ \vdots & b_{2} & \ddots & \ddots & 0 & 0 \\ b_{\alpha-1} & \vdots & \ddots & \ddots & 0 & 0 \\ b_{\alpha} & b_{\alpha-1} & \vdots & \ddots & b_{1} & 0 \\ 0 & b_{\alpha} & \ddots & \vdots & b_{2} & b_{1} \\ 0 & 0 & \ddots & \ddots & \vdots & b_{2} \\ 0 & 0 & 0 & \ddots & b_{\alpha-1} & \vdots \\ 0 & 0 & 0 & 0 & b_{\alpha} & b_{\alpha-1} \\ 0 & 0 & 0 & 0 & 0 & b_{\alpha}\end{array}\right]$

$x=\left[\begin{array}{c}s_{0} \\ s_{1} \\ s_{2} \\ \vdots \\ s_{\alpha-1} \\ s_{\alpha} \\ r_{0} \\ r_{1} \\ r_{2} \\ \vdots \\ r_{\alpha-1} \\ r_{\alpha}\end{array}\right], d=\left[\begin{array}{c}d_{0} \\ d_{1} \\ d_{2} \\ \vdots \\ \vdots \\ \vdots \\ \vdots \\ \vdots \\ \vdots \\ \vdots \\ \vdots \\ d_{2 \alpha+1}\end{array}\right]$ 
Therefore, equation (16) is solved for $x$ and the values of the unknown coefficients $S(z)$ and $R(z)$ are obtained. This system of equations can be solved by means of Matlab, Maple, etc.

The control formulation developed above can be applied to all transfer functions (4), strictly proper. Even when the methodology seems to be just applicable to systems with one pole more than the number of zeros, (see the order of $B(z)$ and $A(z)$ previously defined). This situation is illustrated in Example 1, where some coefficients of $B(z)$ are considered as zero in order to set up the methodology.

\subsection{Internal Stability of Digital Controller}

In order to ensure internal stability of the closedloop system the following particular cases should be taken into account.

Case 1. $C^{\prime}(z)$ is unstable and $R(z)$ is stable. For this case the scheme shown in Figure 2 should be employed. Thus when $R(z)$ is stable the prefilter $\frac{T(z)}{R(z)}$ does not cause closed-loop internal instability in the control scheme shown in Figure 2.

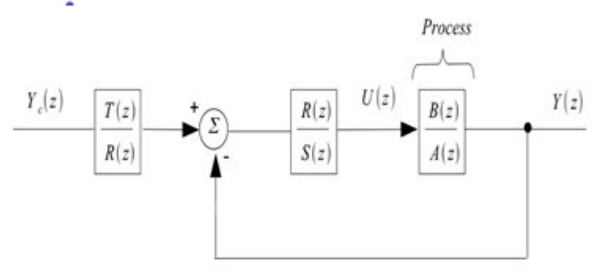

Figure 2. Digital control scheme for the Case 1.

Case 2. $C^{\prime}(z)$ and $R(z)$ are unstable. Here internal stability can neither be guaranteed for the control scheme illustrated in Figure 1, nor for the one in Figure 2. In this case, the polynomial controllers should be designed by considering a different sampling time or a different desired polynomial, $D(z)$. This will be taken into account in Procedure 1, which illustrates on a step-by-step basis how to obtain the aforementioned control scheme. Now, based on the previous discussions and results, the proposed methodology can be summarized as follows.

Procedure 1. To obtain the proposed control scheme.

1. Choose an initial sampling time $T_{d}$, for instance, its value can be chosen to be half the value of the stable or unstable time constant (dominant) of the delay-free system. Then, select $n(n \in \mathrm{N})$ so that $n=\frac{\tau}{T_{d}}$. Compute the sampling time as $T=\frac{\tau}{n}$.

2. Obtain the discrete transfer function representation of the plant.

3. Identify the degrees of $B(z)$ and $A(z)$.

4. Obtain the degrees of polynomials $D(z)$, $R(z)$ and $S(z)$.

5. Propose the desired stable polynomial $D(z)$, where the order of $D(z)$ should be the same as the order of the equation (7).

6. Solve the system of simultaneous linear equations (16) in order to obtain the value of the coefficients $R(z), S(z)$ and $T(z)$.

7. Check the roots of polynomials $C^{\prime}(z)$ and $R(z)$ :

a. If $C^{\prime}(z)$ and $R(z)$ are stable or only $C^{\prime}(z)$ is stable, implement the control scheme shown in Figure 1 and go to step 8 .

b. If $C^{\prime}(z)$ is unstable and $R(z)$ is stable, implement the control scheme shown in Figure 2 and go to step 8 .

c. If $C^{\prime}(z)$ and $R(z)$ are unstable, two options are available:

i. Decrease the sampling time and go to step 2.

ii. Propose a new desired polynomial $D(z)$ and go to step 6 .

8. Use the obtained controllers and verify how they perform via simulation

\section{Examples}

In this section the proposed digital control methodology is applied to different processes in order to illustrate its effectiveness.

Example 1. Let us consider a stable process with time-delay,

$$
\frac{Y(s)}{U(s)}=\frac{1}{s+1} e^{-s} \text {. }
$$

For the discrete time representation of the process expressed by (17) a sampling period $T=0.5$ is used. Thus, the discrete transfer function is given by

$$
\begin{aligned}
G_{d}(z) & \approx\left(\frac{N(z)}{M(z)}\right) z^{-n}=\left(\frac{0.39347}{z-0.6065}\right) \frac{1}{z^{2}}, \\
& =\frac{0.39347}{z^{3}-0.6065 z^{2}}=\frac{B(z)}{A(z)}
\end{aligned}
$$


where $b_{1}=b_{2}=0, \quad b_{3}=0.39347 \quad$ and $a_{1}=-0.6065 \quad a_{2}=a_{3}=0$. The degrees of the polynomials $D^{0}(z), R(z)$ and $S(z)$ are obtained from (14), as

$$
\begin{gathered}
\operatorname{deg}(D(z))=2 \operatorname{deg}(A(z))+1=7 \\
\operatorname{deg}(R(z))=\operatorname{deg}(A(z))=3, \\
\operatorname{deg}(S(z))=\operatorname{deg}(A(z))+1=4
\end{gathered}
$$

Therefore one should propose seven poles for the desired polynomial $D(z)$. Thus the location of the closed-loop poles is proposed as $\{0.1,0.2,0.3,0.1,0.2,0.3,0.1\}$. Then, the polynomials can be written as

$$
\begin{gathered}
D(z)=z^{7}-1.3 z^{6}+0.7 z^{5}-0.202 z^{4}+0.0337 z^{3} \\
-0.00325 z^{2}+0.000168 z-0.0000036 \\
R(z)=r_{0} z^{3}+r_{1} z^{2}+r_{2} z+r_{3} \\
S(z)=(z-1)\left(s_{0} z^{3}+s_{1} z^{2}+s_{2} z+s_{3}\right) \\
T(z)=r_{0}+r_{1}+r_{2}+r_{3}
\end{gathered}
$$

Finally, based on the parameters given in equations (18) and (19), equation (16) is solved for $\mathbf{x}$. The values of the coefficients $R(z), S(z)$ and $T(z)$ are obtained,

$$
\begin{gathered}
R(z)=1.4418 z^{3}-0.86119 z^{2}+4.2697 \times 10^{-4} z \\
-9.1494 \times 10^{-6} \\
S(z)=(z-1)\left(z^{3}+0.3065 z^{2}+0.58589 z+0.55334\right) . \\
S(z)=z^{4}-0.6934 z^{3}+0.2793 z^{2}-0.0325 z-0.5533 \\
T(z)=0.5810
\end{gathered}
$$

In this example the polynomials $R(z)$ and $C^{\prime}(z)$ are stable, therefore the scheme in Figure 1 is used. Figure 3 shows the performance of the digital control methodology subject to an unit step in the reference at $t=0 \mathrm{sec}$ and a disturbance step-signal acting at $t=20 \mathrm{sec}$ with a magnitude of 0.05 units.

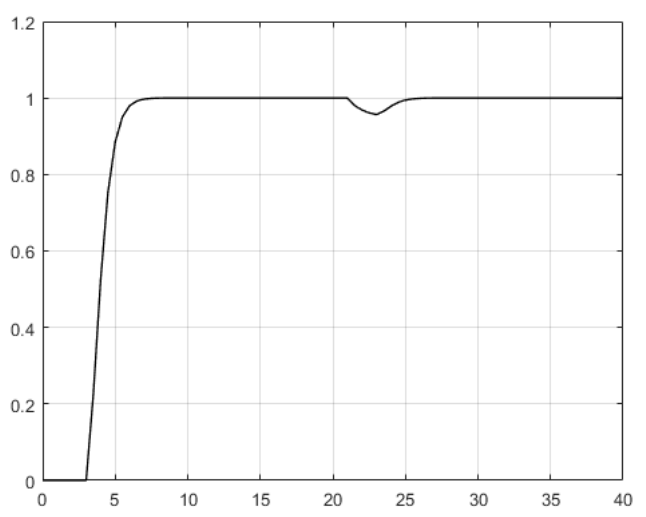

Figure 3. Output response of the system (17) with the proposed digital controller.
Example 2. Let us consider an integrating process with time-delay,

$\frac{Y(s)}{U(s)}=\frac{1}{s(s+1)} e^{-4 s}$.

This example is used in (García \& Albertos, 2013), where an equivalent scheme to the Smith predictor called GP with two stable filters and a PID controller is proposed. As it can be proved, the GP has performed substantially better than many other previous approaches, thus the proposed control methodology will be compared with the GP control strategy. Now for comparison purposes a suitable sampling period, such as $T=0.2$ is considered for both strategies. For this example certain requirements such as a $0 \%$ overshoot and recovery time of $60 s$ for step-type load disturbance are proposed by (García \& Albertos, 2013) and these design specifications have been taken into account. Based on the methodology proposed in this work, the discrete representation of the process expressed by (20) is given by,

$G_{d}(z) \approx\left(\frac{N(z)}{M(z)}\right) z^{-n}=$

$\left(\frac{0.018731(z+0.9355)}{(z-1)(z-0.8187)}\right) \frac{1}{z^{20}}=\frac{B(z)}{A(z)}$

According to the requirements from (García \& Albertos, 2013), one proposed to locate a dominant pole in 0.975 and the leftover poles in 0.1 . Based on these considerations, the equation (16) is solved for $\mathbf{x}$. For this example only the polynomial $R(z)$ is stable, therefore the scheme shown in Figure 2 is employed in order to ensure internal stability of the closed-loop system.

The controller parameters peculiar to the GP (García \& Albertos, 2013) were obtained as the authors have suggested $\lambda=0, F_{1}(z)=\Phi(z, \lambda) \Gamma(z, \lambda)$, where $\Gamma(z, \lambda)=\frac{0.018731(z+0.9355)}{z}$ and $\Phi(z, \lambda)=c(\lambda) \sum_{i=1}^{d} A^{i-1} b z^{-1}$ with $d=20$ and $(A, b, c)$ is a minimum-order state-space model from $\tilde{G}(z, \lambda)=\frac{z}{(z-1)(z-0.8187)} . \quad F_{2}(z)=\frac{\tilde{N}^{*}(z, \lambda)}{(z-\lambda)^{m}}$, where $m$ is the number of zeros of the polynomial $N(z) \cdot \widetilde{N}^{*}(z, \lambda)$ can be obtained from

$\tilde{G}^{*}(z, \lambda)=c(z I-A)^{-1} A^{d} b=\frac{\tilde{N}^{*}(z, \lambda)}{D(z)}$.

Then, the PID controller for the delay-free plant given in (García \& Albertos, 2013) is $K(s)=\frac{0.21(s+1)(s+1 / 20)}{s(0.1 s+1)}$. The discrete time 
controller $K(z)$ is obtained by discretizing $K(s)$. Finally $z^{-d}=z^{-20}$ and $F_{k}(z)=1$.

The simulation results for the two aforementioned control strategies are provided in Figure 4. It can be seen that the proposed methodology improves the system performance for both, the set point tracking and load disturbance rejection. It should be emphasized that the control structure presented in this work is simpler than the control strategy proposed by (Garcia \& Albertos, 2013), if one takes into consideration that this new approach does not use any structure prediction to design the control law.

Moreover, the order of the controller involved in (Garcia \& Albertos, 2013) reach up to $230^{\text {th }}$ order meanwhile the order of the controllers related to the proposed methodology is of $45^{\text {th }}$ order.

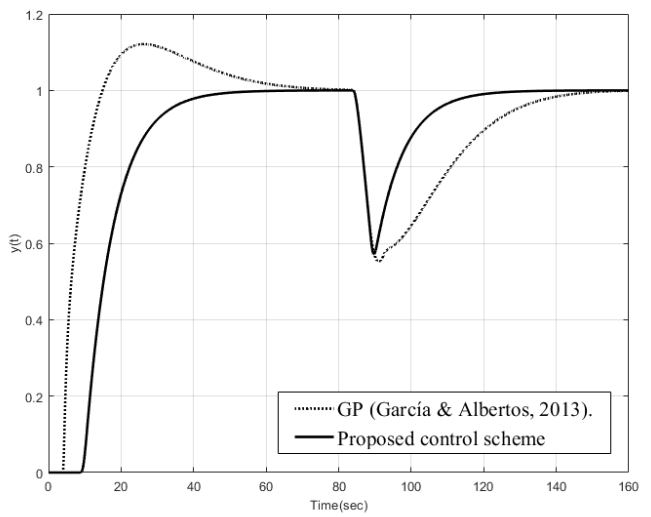

Figure 4. Comparative output response of the system (20) with a step disturbance acting at $\mathrm{t}=80 \mathrm{~s}$. with a magnitude of 0.1 units.

The corresponding control signals are shown in Figure 5. It can be observed that the control signal obtained by employing the proposed method shows a smooth variation in comparison with the one obtained by using the approach proposed by (García \& Albertos, 2013).

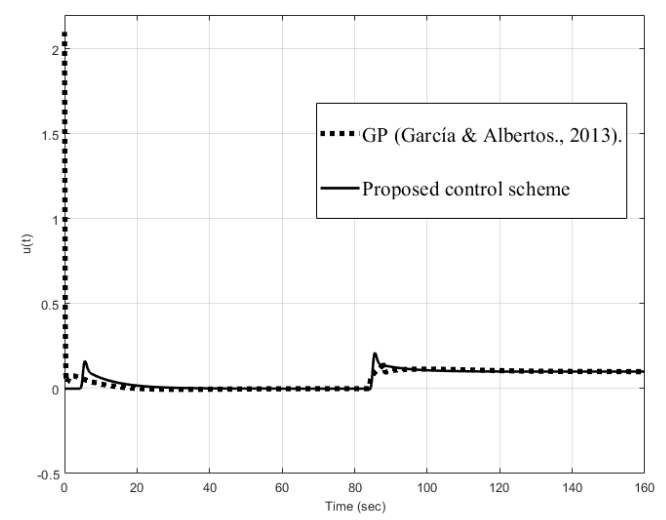

Figure 5. Control signal of the system
Example 3. Let us consider a triple integrating process with time-delay,

$$
\frac{Y(s)}{U(s)}=\frac{1}{s^{3}} e^{-4 s} .
$$

In this example a sampling period of $T=0.2$ is considered. Thus, $B(z)$ and $A(z)$ are obtained through the discretization of the process in (22), then the degrees of the controllers are calculated. This experiment shows the output performance of the system involved based on the position of two different closed-loop poles location, i.e, two different desired polynomials $D(z)$. In the first case, all poles of the desired polynomial $D(z)$ are located at 0.2 . In the second case, the dominant pole has been located at 0.94 and the other poles at 0.2 . In this example only the polynomial $R(z)$ is stable in both locations, therefore the scheme in Figure 2 is used. Figure 6 shows the output performance response from the proposed digital methodology when a step disturbance acts at $t=$ $50 s$ with a magnitude of 0.005 units.

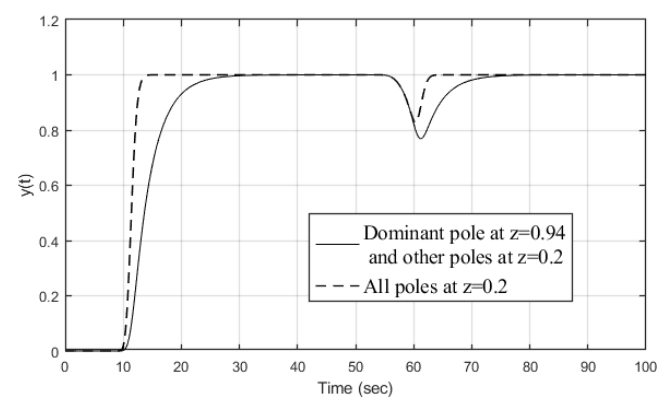

Figure 6. Output response of the system (22) with the proposed digital controller and different locations

Example 4. Let us consider an unstable process with large time-delay,

$$
\frac{Y(s)}{U(s)}=\frac{1}{s-1} e^{-5 s} \text {. }
$$

In this case, a sampling period $T=0.1$ is used. The dominant pole has been located at 0.95 and the leftover poles at 0.1 . In this example the scheme in Figure 2 is used, as only the polynomial $R(z)$ is stable. It is important to highlight that this system cannot be stabilized by the proposed strategy from (Márquez-Rubio et al., 2015), since its stability condition cannot be satisfied ( $\tau<4 / a$ with $\tau=5$ and $a=1$ ). The delayed system (23) is stabilized in (Fragoso-Rubio et al, 2019) using a prediction-observer scheme with the parameters $m=20$ (which implies an observer of 20th order), 
$L=1.5$ and $k=-1.1$. Figure 7 shows the output performance of both strategies. It can be noticed that the results are similar.

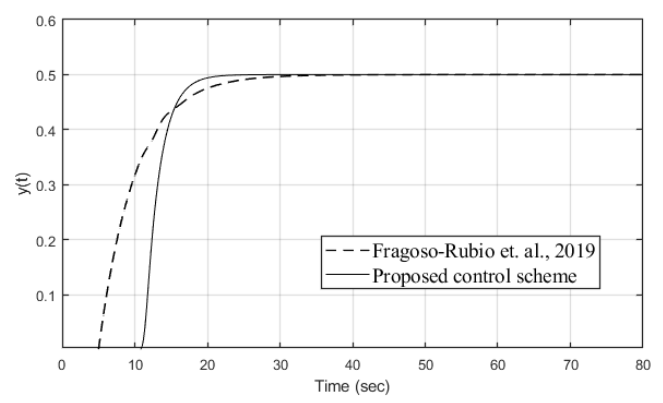

Figure 7. Output response of the system (23) with the digital controller.

\section{Conclusion}

This work proposes a digital control methodology for systems with large time

\section{REFERENCES}

Fragoso-Rubio, V., Velasco-Villa, M., HernándezPérez, M. A., Del Muro-Cuéllar, B. \& MárquezRubio, J. F. (2019). Prediction-observer scheme for linear systems with input-output time-delay, International Journal of Control, Automation and Systems, 17(8), 2012-2025.

Gang-Gyoo, J. \& Yung-Deug, S. (2019). Design of a Nonlinear PID controller and tuning rules for first-order plus time delay models, Studies in Informatics and Control, 28(2), 157-166. DOI: 10.24846/v28i2y201904

García, P. \& Albertos, P. (2008). A new dead-time compensator to control stable and integrating process with long dead-time, Automatica, 44(4), 1062-1071.

García, P. \& Albertos, P. (2013). Robust tuning of a generalized predictor-based controller for integrating and unstable systems with long timedelay, Journal of Process Control, 23, 1205-1216.

Hagglund, T. (1996). An industrial dead-time compensating PI controller, Control Engineering Practice, 4, 749-756.

Liu, T., Zhang, W. \& Gu, D. (2005). Analytical design of two-degree-of-freedom control scheme for open-loop unstable processes, Journal of Process Control, 15, 559-572. delay processes. The proposed methodology can be applied to all kinds of systems (stable, integrating and unstable) with time delays. It is based on a discrete time representation of the dynamics of the system involved and uses a polynomial approach. By employing it the problems of step reference tracking and step disturbance rejection are also solved. It should be mentioned that the sampling period is an important parameter for this methodology, as the degree of the controllers depends on it. In order to ensure internal stability of the closedloop system two different implementations are presented. The two implementations are based on the stability of the polynomials $C^{\prime}(z)$ and $R(z)$. Numerical simulations show improved results with respect to recent control strategies in literature, in terms of stability, step tracking and rejecting step disturbances.

Márquez-Rubio, J. F., Del-Muro-Cuéllar, B., Velasco-Villa, B. \& Alvarez-Ramírez, J. J. (2015). An improved sufficient condition for stabilisation of unstable first-order processes by observer-state feedback, International Journal of Control, 88(2), 403-412.

Owens, D. H. \& Raya, A. (1982). Robust stability of Smith Predictor controllers for time-delay systems. IEE Proceedings D- Control Theory and Applications, 129(6), 298-304.

Palmor, Z. (1996). Time-delay compensation: Smith Predictor and its modifications, The Control Handbook, 1, 224-229.

Richard, J. P. (2003). Time-Delay Systems: An Overview of Some Recent Advances and Open Problems, Automatica, 39, 1667-1694.

Seshagiri, A. R. \& Chidambaram, M. (2012). PI/PID controllers design for integrating and unstable systems. In Vilanova R., Visioli A. (eds) PID Control in the third millennium, Advances in Industrial Control, 75-111. Springer, London.

Visioli, A. \& Zhong, Q. (2011). Control of integral processes with dead time, Advances in Industrial Control, 9-47. Springer, London. 